\title{
Fluorescent Monitoring of Photodynamic Therapy for Skin Cancer in Clinical Practice
}

\author{
DOI: $10.17691 /$ stm2015.7.2.10
}

Received November 15, 2014

S.V. Gamayunov, PhD, Head of Photodynamic Therapy Department'; Assistant Professor, Department of Oncology,

Postgraduate Faculty2; Senior Researcher ${ }^{3}$;

E.V. Grebenkina, PhD, Oncologist, Photodynamic Therapy Department';

A.A. Ermilina, Technologist, Department of Radiophysical Methods in Medicine4;

V.A. Karov, Oncologist, Photodynamic Therapy Department';

K. König, PhD, Leading Scientist Femtosecond Biophotonics Laboratory3; Full Professor ${ }^{5}$; CEO6;

K.S. Korchagina, Resident Physician, Department of Pathological Anatomy ${ }^{7}$;

R.R. Skrebtsova, Oncologist, Photodynamic Therapy Department';

V.M. Terekhov, PhD, Chief Doctor';

I.G. Terentiev, MD, DSc, Professor, Head of the Department of Oncology, Postgraduate Faculty2;

I.V. Turchin, PhD, Head of the Department of Radiophysical Methods in Medicine; ${ }^{4}$ Senior Researcher ${ }^{3}$;

N.M. Shakhova, MD, DSc, Leading Researcher, Department of Radiophysical Methods in Medicine ${ }^{4}$;

Leading Researcher, Femtosecond Biophotonics Laboratory ${ }^{3}$

${ }^{1}$ Nizhny Novgorod Regional Oncologic Hospital, 190, bld. 5 Rodionova St., Nizhny Novgorod,

603000, Russian Federation;

${ }^{2}$ Nizhny Novgorod State Medical Academy, 10/1 Minin and Pozharsky Square, Nizhny Novgorod,

603005, Russian Federation;

${ }^{3}$ Lobachevsky State University of Nizhni Novgorod, 23 Prospekt Gagarina, Nizhny Novgorod, Russian Federation,

603950;

${ }^{4}$ Institute of Applied Physics, Russian Academy of Sciences, 46 Ulianova St., Nizhny Novgorod, 603950,

Russian Federation;

${ }^{5}$ Saarland University, Campus A5.1 Saarbrücken, 66123, Germany;

${ }^{6}$ JenLab GmbH, 1 Schillerstraße, Jena, 07745, Germany;

${ }^{7}$ Moscow State Medical Stomatological University named after A.I. Evdokimov, 20, bld. 1 Delegatskaya St.,

Moscow, 127473, Russian Federation

The aim of the investigation was to assess the fluorescent imaging capabilities to monitor photodynamic therapy (PDT) of non-melanoma skin cancer, and study the correlation of PS (photosensitizer) fluorescence value (accumulation and photobleaching rate) and efficacy of the treatment provided.

Materials and Methods. The study was conducted in the Nizhny Novgorod Regional Oncologic Hospital. We analyzed fluorescent images and PDT outcomes in 226 patients with non-melanoma skin carcinomas.

Results. The assessment of short-term treatment results revealed the relationship between PS photobleaching and tumor complete response rate: a complete response was found in $89 \%$ cases in complete photobleaching, in $87 \%$ cases — in partial photobleaching, and in $81 \%$ - in no photobleaching $(p>0.05)$. However, we found no effect of PS accumulation rate on complete response rate. The analysis of long-term results with significant difference $(p=0.044)$ showed tumor recurrence rate in low PS concentration (tumor/norm) to be $9.5 \%$, while in moderate and high concentrations the recurrence rate appeared to be $4.1 \%$. There was revealed the tendency for recurrence rate increase $-10.4 \%$ with no PS photobleaching versus $4.4 \%$ in PS complete and partial photobleaching $(\mathrm{p}=0.051)$. The patients with high accumulation rate and complete PS photobleaching had the best clinical findings, the observation period being from 4 to 40 months.

Conclusion. Fluorescent monitoring enables to maintain noninvasive control of PS accumulation and photobleaching that can contribute to the selection of individual laser exposure parameters. It is reasonable to develop multimodal bioimaging for follow-up real-time monitoring of basic photodynamic reactions and treatment results.

Key words: photodynamic therapy monitoring; PDT; photosensitizer; fluorescent diagnostics.

Photodynamic therapy (PDT) is a modern, minimally invasive technique of cancer treatment, which currently has a widespread application in clinical practice [1-3]. The basic components of photodynamic reaction are a photosensitizer (PS) pre-administered and selectively accumulated in tumor tissue, the light of an appropriate wavelength corresponding to maximum PS absorption, and oxygen [2, 4, 5]. PDT can be used as curative therapy for early neoplastic processes, as well as in schemes of palliative therapy in advanced

For contacts: Gamayunov Sergey Victorovich, e-mail: Gamajnovs@mail.ru 
tumors as an independent technique or combined with other approaches [1-3]. PDT is an attractive technique for clinical application in oncology due to the following factors: minimal damage of surrounding normal tissues due to selectively accumulation PS in a tumor, insignificant systemic effects, the lack of the mechanisms of primary and acquired resistance, no limiting cumulative PS doses and light exposure, and therefore, capability of repeated procedures. Additional advantages are availability in an out-patient setting, high tolerability with minimal pain reactions, compatibility with other treatment techniques, good cosmetic results. The factors limiting a wide application of PDT are the following: laser intensity attenuation with light penetration depth in biotissues, and subsequently, efficacy decrease and unpredictability of photodynamic reactions; the effect of tumor blood supply and oxygenation on the procedure results; the absence of morphological control of treatment; empirical matching of the modes of exposure [1-5]. The effect of these drawbacks on PDT results can be minimized by using effective methods to assess the therapy adequacy. To achieve success in follow-up control and the control of photodynamic reaction, monitoring techniques should be noninvasive and quick-action with real-time mode $[5,6]$. One of such techniques is fluorescent imaging, which enables to assess PS accumulation rate in a tumor, specify the lesion extent and control PS photobleaching. Based on fluorescent diagnosis data, laser exposure mode can be optimized: procedure time after PS administration, laser power density and dose [5, 7-11].

The aim of the investigation was to assess the fluorescent imaging capabilities to monitor photodynamic therapy of non-melanoma skin cancer, and study the correlation of PS fluorescence value (accumulation and photobleaching rate) and efficacy of the treatment provided.

Materials and Methods. The survey was conducted on the basis of Nizhny Novgorod Regional Oncologic Hospital. We monitored fluorescence images and assessed PDT findings in 226 patients with basal cell and squamous cell skin carcinomas.

The present study was approved by the Ethics Committee of Nizhny Novgorod State Medical Academy, and complies with the declaration of Helsinki (adopted in June, 1964 (Helsinki, Finland) and revised in October, 2000 (Edinburg, Scotland)). Written informed consent was obtained from all patients.

Fotoditazin (Veta-grant, Russia), a chlorine preparation, was systemically injected as a PS, $1.0 \mathrm{mg} / \mathrm{kg}$ body mass. The patients were exposed to laser radiation by Lakhta-Milon (Milon Laser, Russia), wavelength of radiation being $662 \mathrm{~nm}$. For light delivery to a tumor we used optical fibers with micro- and macrolens. Power density averaged $0.3 \mathrm{~W} / \mathrm{cm}^{2}\left(0.28-0.32 \mathrm{~W} / \mathrm{cm}^{2}\right)$, energy density - $200 \mathrm{~J} / \mathrm{cm}^{2}$ in basal cell carcinoma, and $300 \mathrm{~J} / \mathrm{cm}^{2}$ - in squamous cell carcinoma.
PS fluorescence parameters were analyzed using Fluovisor (Atcus Company, Russia). 1.5-2.0 h after PS injection (maximum accumulation of preparation according to Fotoditazin pharmacodynamics) we assessed fluorescence intensity in reference points of a tumor $\left(I_{t 0}\right)$ and surrounding intact skin $\left(I_{N 0}\right)$ determining fluorescence intensity tumor/norm relationship $\left(I_{t_{0}} / I_{N 0}\right)$. Photobleaching of the preparation $\left(\Delta I_{t} / I_{N}\right)$ was calculated after having assessed fluorescence in reference points immediately after the laser exposure according to the following formula:

$$
\Delta \frac{I_{t}}{I_{N}}=\left[\frac{I_{t}}{I_{N}}\right] /\left[\frac{I_{t 0}}{I_{N 0}}\right]
$$

Fluorescence intensity indirectly indicates PS concentration in a tumor.

PDT results were clinically assessed based on international criteria RECIST [12] by changing the two highest parameters followed by biopsy for morphological verification. Side effects were classified by CTCAE v. 4.03 [13]. Follow-up period varied from 2 to 40 months, median -26 months.

Results and Discussion. The obtained data on PS accumulation grade (concentration) in a tumor in relation to healthy tissues enabled to distinguish three groups of cases. Group 1 (conditionally assessed as "no accumulation") was characterized by low ratio of tumor/norm concentration $I_{t} I_{N}<1.1$, when fluorescence of surrounding tissues and the tumor were comparable. If $I_{t} / I_{N}>1.3$, "high accumulation" was diagnosed. Fluorescence parameters being within the range of $1.1<I_{t} / I_{N}<1.3$ were referred to "low accumulation" (Figure 1).

The assessment of PS photobleaching grade immediately after laser exposure enabled to distinguish three groups of reactions as well (Figure 2). If fluorescence tumor/norm relationship decreased by more than $20 \%$ in relation to the reference level $\left[\left(I_{t} / I_{N}\right) /\left(I_{t 0} / I_{N 0}\right)<0.8\right]$, PS photobleaching was considered complete. Fluorescence tumor/norm relationship decreased by less than $10 \%$ in relation to the reference level $\left[\left(I_{t} / I_{N}\right)\left(I_{t_{0}} I_{N_{N}}\right)>0.9\right]$ corresponded to no PS photobleaching, and the parameters within the range of 0.8-0.9 were considered as partial photobleaching.

Clinically, PDT findings in the patients under study can be assessed as good ones: at median being 26 months, recovery (a recurrence-free period) was found in 198 patients of $226(87.6 \%), 18$ patients (12.4\%) were found to have recurrences, and given repeated PDT procedures with good clinical results, and had 13-month median duration of recurrence-free period after repeated PDT. Significantly, the overwhelming majority of patients $(96 \%)$ under study appeared to have good functional and cosmetic results in (Figure 3).

There were no complications revealed after PDT, however, some patients were found to have adverse events: insignificant photosensitivity increase (2 cases), persistent erythema (5 cases), moderate pain syndrome in 

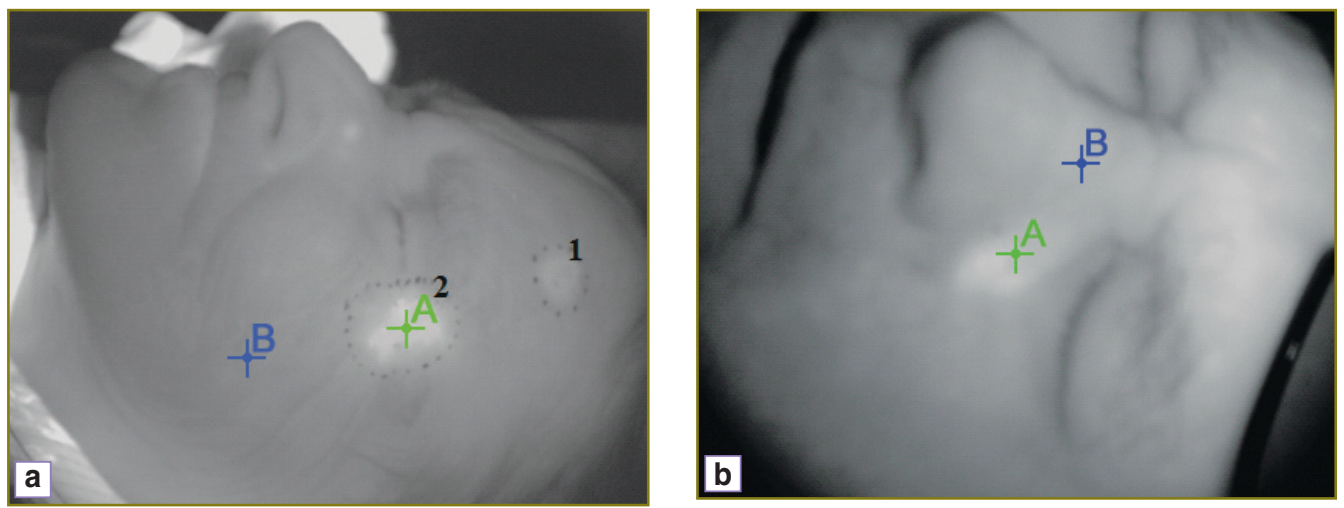

Figure 1. Fluorescent tumor images (dotted lines indicate tumor foci, points $A$ and $B$ corresponding to cursor of fluorescence intensity change). Photosensitizer accumulation grade: (a) patient Ts., multicentric basal cell facial skin carcinoma, no accumulation (area 1), fluorescence in the tumor is comparable with fluorescence of surrounding tissues (ratio less than 1.1), and high accumulation (area 2), fluorescence in the tumor significantly differs from that of surrounding tissues (ratio more than 1.3); (b) patient V., basal cell facial skin carcinoma, low accumulation, fluorescence in the tumor slightly differs from that of surrounding tissues (ratio 1.1-1.3)

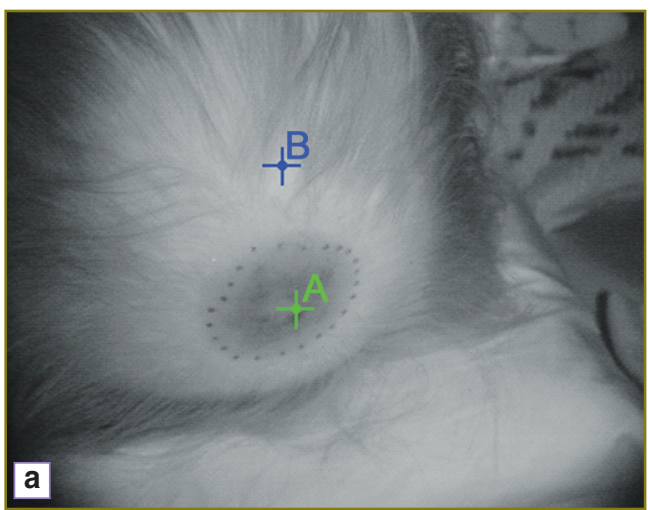

Figure 2. Fluorescent tumor images (dotted lines indicate tumor foci, points $A$ and $B$ corresponding to cursor of fluorescence intensity change). Photosensitizer photobleaching grade: (a) patient $P_{\text {., }}$ basal cell scalp carcinoma, complete photobleaching $\left[\left(I_{t} / I_{N}\right) /\left(I_{t 0} / I_{N 0}\right)<0.8\right]$; (b) patient $Y$., basal cell facial skin carcinoma, partial photobleaching $\left[0.8<\left(I_{t} / I_{N}\right) /\left(I_{t 0} / I_{N 0}\right)<0.9\right]$; (c) patient S., basal cell nasal skin carcinoma, no photobleaching $\left[\left(I_{t} / I_{N}\right) /\left(I_{t 0} / I_{N 0}\right)>0.9\right]$

the exposure area (78 cases), prolonged (over 3 months) inflammatory reactions in the exposure area (37 cases), formation of hypo- and hypertrophic scars (9 cases). All adverse events were assessed as I-II grade according to CTCAE v. 4.03. No side effects or complications related to the monitoring technique were revealed.

The analysis of tumor response to PDT depending on the preparation accumulation grade $\left(I_{t_{0}} / I_{N_{0}}\right)$ showed
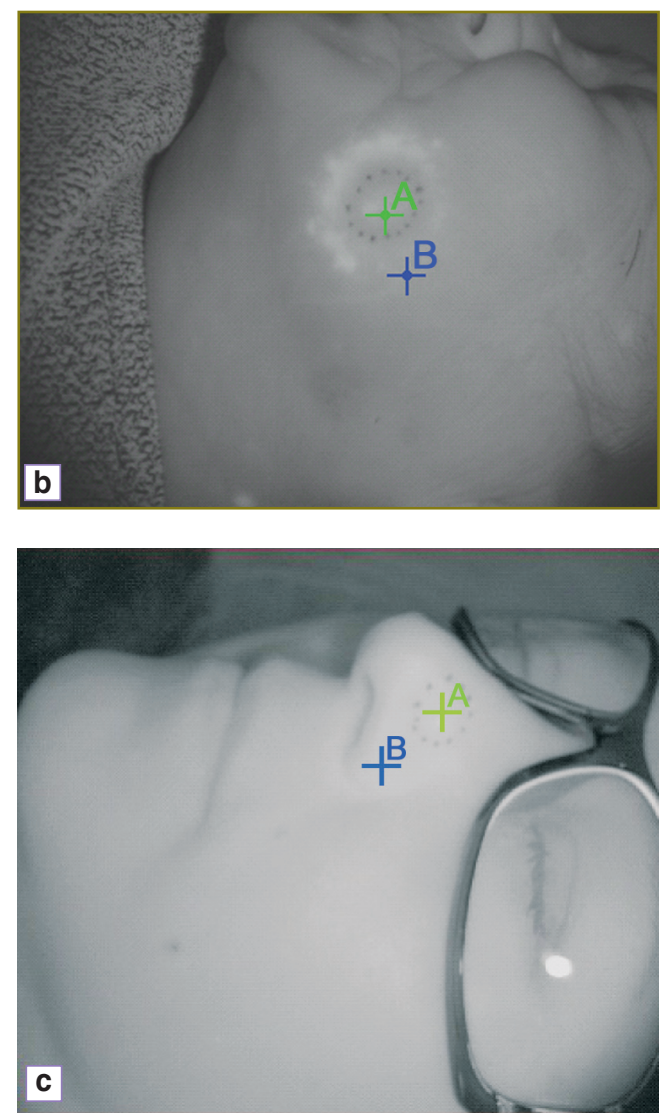

no significant differences: complete response rate was $90-93 \%$ in all accumulation degrees. The analysis of the effect of photobleaching on short-term results $\left(\Delta I_{t} / I_{N}\right)$ showed the tendency for the increase of tumor complete response rate in complete PS photobleaching (See the Table).

The analysis of long-term results showed tumor recurrence rate in no PS accumulation and/or no PS 

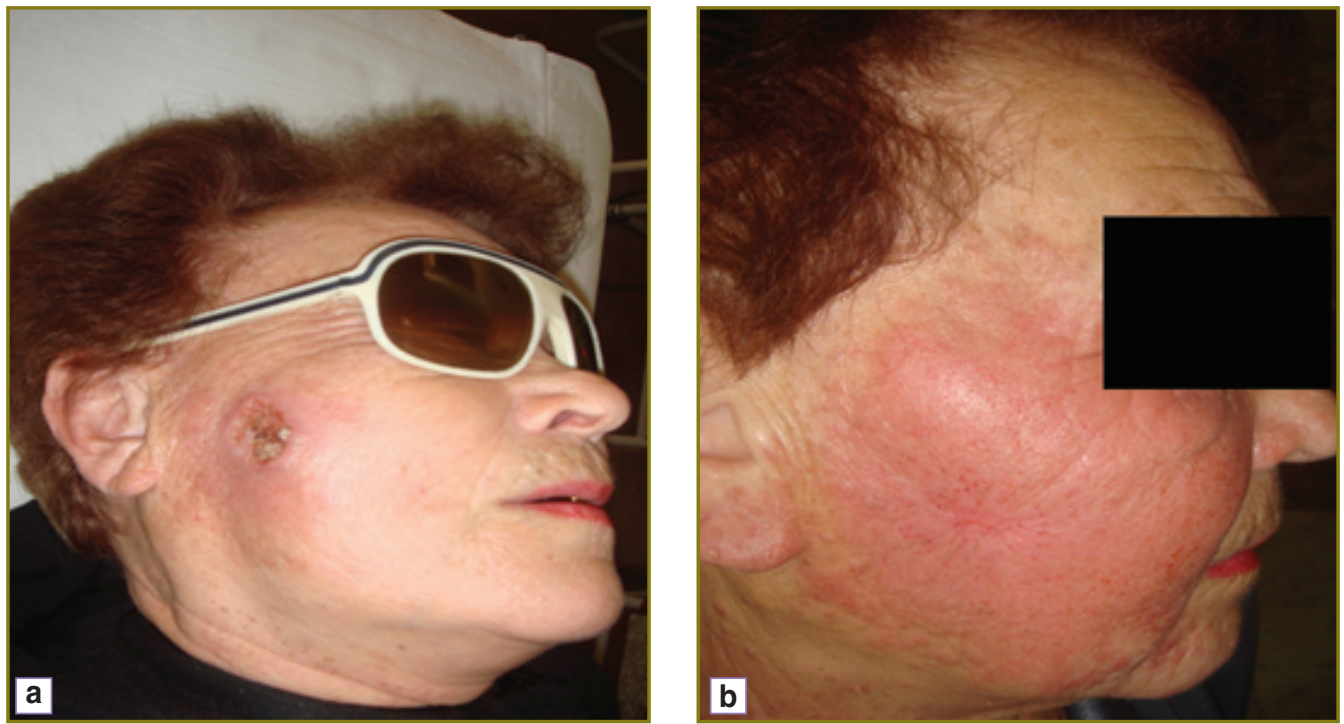

Figure 3. Photo of a 68-year female patient, the diagnosis is "primary basal cell facial skin carcinoma": (a) before PDT, a tumor, $45 \times 48 \mathrm{~mm}$ in size, $6 \mathrm{~mm}$ in thickness (exophytic component and invasion); (b) after PDT, complete tumor response, good cosmetic effect, 4-year recurrence-free period

The dependence of short- and long-term results on fluorescent parameters of a photosensitizer

\begin{tabular}{|lcc|}
\hline \multicolumn{1}{|c}{ Fluorescent parameter } & $\begin{array}{c}\text { Short-term results, } \\
\text { complete tumor } \\
\text { responses (\%) }\end{array}$ & $\begin{array}{c}\text { Long-term results, } \\
\text { recurrence rate (\%) }\end{array}$ \\
\hline $\begin{array}{l}\text { High PS accumulation } \\
I_{t} / I_{N}>1.3\end{array}$ & 90 & 4.1 \\
\hline $\begin{array}{l}\text { Low PS accumulation } \\
1.1<I_{t} / I_{N}<1.3\end{array}$ & 93 & \\
\hline $\begin{array}{l}\text { No PS accumulation } \\
I_{t} / I_{N}<1.1\end{array}$ & 91 & 9.5 \\
\hline $\begin{array}{l}\text { Complete PS photobleaching } \\
\Delta I_{t} / I_{N}<0.8\end{array}$ & 89 & $(p=0.044)$ \\
\hline $\begin{array}{l}\text { Partial PS photobleaching } \\
0.8<\Delta V_{t} / I_{N}<0.9\end{array}$ & 87 & \\
\hline No PS photobleaching & 81 & 4.4 \\
$\Delta I_{t} / I_{N}>0.9$ & $(\mathrm{p}>0.05)$ & 10.4 \\
\hline
\end{tabular}

parameters for the treatment prognosis is promising. According to our data, the patients with low PS accumulation in a tumor and/or no PS photobleaching after PDT run the highest recurrence risk, and require intensive case monitoring. Other authors have reported similar findings in series of experimental studies [5, 7-11].

To improve PDT efficiency in clinical practice it is important to use both passive observation of the main treatment components and also the possibility to plan the regimens and control basic PDT reactions in real time mode. Fluorescent imaging is used as a monitoring technique to detect real tumor borders in order to form optimal fields and boundaries of laser exposure that is particularly relevant if there is an invisible infiltrative component of the tumor or a multicentric lesion. An adequate choice of light exposure field contributes to

photobleaching to be significantly higher than in the presence of fluorescent contrast between a tumor and the surrounding tissues and complete or partial photobleaching.

The best short- and long-term results were found in 62 patients with the combination of high PS accumulation and its complete photobleaching after the procedure. The number of complete tumor responses in this group was $94 \%(58 / 62)$, and there were only $3.2 \%$ recurrences (2/62), the observation period being from 4 to 40 months.

The findings indicate that fluorescent imaging can be used for noninvasive observation of the key points for PDT mechanism: PS accumulation and photobleaching, thus it can be argued that the monitoring of these the decrease of recurrence risk on peripheries and the risk of residual tumors [7, 14].

It seems advisable to study fluorescent parameters to use fluorescence to monitor and optimize the modes of light exposure. In particular, light-drug interval, power density and energy density can be patient-specific depending on PS time and degree of accumulation and photobleaching $[4,5,8,11,15]$. To manage realtime photodynamic responses it is reasonable to use fluorescent imaging just during the light exposure [8] that was, unfortunately, impossible in our study due to technological imperfection of the equipment used. One more restriction of the study was to measure fluorescence in conventional units, and analysis of relative values 
that enabled to give only indirect estimation of true PS concentration in a tumor. On the other hand, relative values enabled to minimize measurement errors related to personal characteristics of biotissues and the significant effect of the external study environment on the results. All the above mentioned indicate the necessity to improve equipment for PDT fluorescence monitoring.

For widespread use in clinical practice a diagnostic system should have the reproducibility of results that is achieved by minimization of the effect of internal equipment noise and external factors (background light, the distance from equipment to a patient) on measurement data. For a quantitative assessment of PS concentration in a tumor it is necessary to consider individual optical characteristics of biotissues (scattering and absorption parameters including heterogeneity of an object), since these characteristics influence the level of the measured fluorescence. Considering that these parameters vary from patient to patient, as well as significantly change during PDT, additional measurements and corresponding mathematical processing are required for their correct consideration. These measurements can be presented as both by point [10,16], and by means of digital cameras using several radiation sources and receiving spectral channels $[17,18]$. It should be noted that PDT efficiency decreases with the depth, since an optical field exponentially drops with depth. If we know tumor depth, optical characteristics of tissue and PS accumulation rate in a tumor, we can assess an optical radiation dose [19, 20].

It should be noted that PDT mechanism is based on the interaction of the main components (a photosensitizer, light, oxygen) and realized in three types of responses: fluorescence phenomenon, photochemical reaction I (oxygen-free) and II (with oxygen) [4, 5]. Fluorescent imaging enables to control only PS parameters through the observation of fluorescence phenomenon, while the basic component of PDT mechanism is type II photochemical reaction with singlet oxygen formed resulting in a direct cytotoxic action and ablation of tumor vascular bed. Other approaches are reasonable to be used to monitor these PDT elements. A series of studies $[15,21,22]$ has shown the significance of the diagnostics of tumor oxygen status before PDT and the monitoring of singlet oxygen formation during therapy using the combination of spectroscopy methods to assess reference tissue oxygenation, fluorescence techniques to control PS accumulation and the techniques to detect singlet oxygen luminescence after exposure.

Another direction of optical imaging is based on the use of optical coherence tomography (OCT) for noninvasive diagnostics of the changes of tumor structures after PDT. By an example of non-melanoma skin cancer there has been shown OCT efficiency in imaging tumor borders for PDT planning, in the assessment of short-term (tumor response to therapy, adequacy of healing processes) and long-term (diagnosis of residual foci) results [23, 24]. New OCT modifications are informative in relation to tumor microcirculation that is prospective for monitoring of the main pathophysiological PDT mechanism ablation of tumor bloodstream $[25,26]$.

An alternative and rather promising approach for noninvasive PDT monitoring is the development of opticoacoustic imaging technology. This type of bioimaging combining the advantages of optical and acoustic techniques is growing rapidly in recent years [27]. First findings have shown that optico-acoustic imaging in PDT enables to obtain data on tumor structure and architectonics of its microvascular surrounding before therapy, as well as the change of object morphology after PDT, and can be also used to monitor one of the most critical components of photodynamic responses parameters of tumor oxygenation [28].

One more type of noninvasive monitoring is multiphoton imaging, its instrumental realization and introduction in clinical practice have become possible due to the femtosecond optics development [29-31]. These techniques are certain to be promising for tumor detection. The study [29] showed the advantages of multiphoton tomography compared to confocal microscopy for in vivo diagnostics of basal cell skin cancer. The capability of noninvasive dynamic detection of decreased fluorescence of reduced nicotine-amideadenine-dinucleotide in skin in experimental arterial occlusive disease makes multiphoton tomography a promising monitoring technique to control the methods having an effect on microcirculation including PDT control [30].

All the above mentioned approaches have certain advantages and drawbacks. The review by König [31] has shown the advisability of combination of various techniques: both in instrumental realization of hybrid technique, and complementary use of different technologies.

A large-scale implementation of the present technique in clinical practice requires an improved instrumental basis of PDT fluorescent monitoring, the development of methods of its usage, and pursuance of randomized studies on effective application of this technique in clinical practice. The technique development can promote personification of PDT due to the planning of individual parameters of laser exposure, and realtime control of photodynamic reactions depending on fluorescence parameters that will make it possible to achieve maximum number of complete tumor responses to therapy and reduce recurrence rate. The development of multimodal bioimaging based on fluorescent imaging, new OCT modifications, optico-acoustic and multiphoton imaging is prospective and relevant for further development of health monitoring and individualization of PDT parameters.

Conclusion. By the example of photodynamic therapy of skin carcinoma using a Chlorine e6 photosensitizer 
we have shown fluorescent imaging to be an effective noninvasive technique to assess the characteristics of a photosensitizer - one of the basic components of photodynamic reactions. The monitoring of such parameters as PS accumulation and photobleaching enables to predict PDT results.

Study Funding. The study was carried out within Complex PDT Development and Implementation Program with financial support of experimental works from Russian Foundation for Basic Research (Grant of Russian Foundation for Basic Research No.14-0200753/14), Lobachevsky State University of Nizhni Novgorod Competitive Growth Program (Agreement between Ministry of Education and Science of the Russian Federation and Lobachevsky State University of Nizhni Novgorod dated August, 27, 2013, No.02. B.49.21.0003), and within the frame of technological developments and clinical studies of Ministry of Education and Science of the Russian Federation (Project RFMEFI60414X0027, Contract No.14. B25.31.0015).

Conflict of interests. The authors have no conflict of interests to declare.

\section{References}

1. Foley P., Freeman M., Menter A., Siller G., El-Azhary R.A., Gebauer K., et al. Photodynamic therapy with methyl aminolevulinate for primary nodular basal cell carcinoma: results of two randomized studies. Int $J$ Dermatol 2009 Nov; 48(11): 1236-1245, http://dx.doi.org/10.1111/ j.1365-4632.2008.04022.x.

2. Agostinis P., Berg K., Cengel K.A., Foster T.H., Girotti A.W., Gollnick S.O., et al. Photodynamic therapy of cancer: an update. CA Cancer J Clin 2011 Jul-Aug; 61(4): 250-281, http://dx.doi.org/10.3322/caac.20114.

3. Sokolov V.V., Karpova E.S., Filonenko E.V., Belous T.A., Frank G.A. Successful combination of endoscopic treatment (photodynamic therapy + electrocoagulation) of a patient with early lower third esophageal cancer (14-year clinical observation). Fotodinamicheskaya terapiya $i$ fotodiagnostika 2013; 1: 3-6.

4. Allison R.R., Moghissi K. Photodynamic therapy (PDT): PDT mechanisms. Clin Endosc 2013 Jan; 46(1): 24-29, http:// dx.doi.org/10.5946/ce.2013.46.1.24.

5. Celli J.P., Spring B.Q., Rizvi I., Evans C.L., Samkoe K.S., Verma S., et al. Imaging and photodynamic therapy: mechanisms, monitoring, and optimization. Chem Rev 2010 May; 110(5): 2795-2838, http://dx.doi.org/10.1021/ cr900300p.

6. Kalugina R.R., Yanvareva I.A., Strel'tsova Yu.A., Gamayunov S.V., Slugarev V.V., Denisenko A.N., et al. Noninvasive monitoring of pathophysiological processes at different photodynamic therapy stages. Sovremennye tehnologii $v$ medicine 2009; 1: 107-110.

7. Chissov V.I., Filonenko E.V., Reshetov I.V., Zaytsev A.M., Loshakov V.A., Kurzhupov M.l., et al. Intraoperative fluorescent diagnostics and photodynamic therapy in patients with brain metastatic lesions. Rossiyskiy onkologicheskiy zhurnal 2011; 2: 4-7.
8. Kruijt B., van der Ploeg-van den Heuvel A., de Bruijn H.S., Sterenborg H.J., Amelink A., Robinson D.J. Monitoring interstitial $\mathrm{m}$-THPC-PDT in vivo using fluorescence and reflectance spectroscopy. Lasers Surg Med 2009 Nov; 41(9): 653-664, http://dx.doi.org/10.1002/lsm.20845.

9. Wang Y., Gu Y., Liao X., Chen R., Ding H. Fluorescence monitoring of a photosensitizer and prediction of the therapeutic effect of photodynamic therapy for port wine stains. Exp Biol Med 2010 Feb; 235(2): 175-180, http://dx.doi.org/10.1258/ ebm.2009.009294.

10. Brydegaard M., Haj-Hosseini N., Wardell K., Andersson-Engels S. Photobleaching-insensitive fluorescence diagnostics in skin and brain tissue. IEEE Photonics J 2011 Jun; 3(3): 407-421, http://dx.doi. org/10.1109/jphot.2011.2141656.

11. Ascencio M., Collinet P., Farine M.O., Mordon S. Protoporphyrin IX fluorescence photobleaching is a useful tool to predict the response of rat ovarian cancer following hexaminolevulinate photodynamic therapy. Lasers Surg Med $2008 \mathrm{Jul} ; 40$ (5): 332-341, http://dx.doi.org/10.1002/lsm.20629.

12. Shanbhogue A.K., Karnad A.B., Prasad S.R. Tumor response evaluation in oncology: current update. $J$ Comput Assist Tomogr 2010 Jul; 34(4): 479-484, http://dx.doi. org/10.1097/RCT.0b013e3181db2670.

13. Common terminology criteria for adverse events (CTCAE). Version 4.03. U.S. Department of Health and Human Services, National Institutes of Health, National Cancer Institute; 2010 June 14.

14. Kruijt B., van der Snoek E.M., Sterenborg H.J., Amelink A., Robinson D.J. A dedicated applicator for light delivery and monitoring of PDT of intra-anal intraepithelial neoplasia. Photodiagnosis Photodyn Ther 2010 Mar; 7(1): 3-9, http://dx.doi.org/10.1016/j.pdpdt.2010.01.006.

15. Jarvi M.T., Patterson M.S., Wilson B.C. Insights into photodynamic therapy dosimetry: simultaneous singlet oxygen luminescence and photosensitizer photobleaching measurements. Biophys J 2012 Feb; 102(3): 661-671, http:// dx.doi.org/10.1016/j.bpj.2011.12.043.

16. Xie H., Xie Z., Mousavi M., Bendsoe N., Brydegaard M., Axelsson J., Andersson-Engels S. Design and validation of a fiber optic point probe instrument for therapy guidance and monitoring. J Biomed Opt 2014 Mar; 19(7): 71408, http:// dx.doi.org/10.1117/1.JBO.19.7.071408.

17. Valdes P.A., Jacobs V.L., Wilson B.C., Leblond F., Roberts D.W., Paulsen K.D. System and methods for widefield fluorescence imaging during neurosurgery. Opt Lett 2013 Aug; 38(15): 2786-2788, http://dx.doi.org/10.1364/ ol.38.002786.

18. Sexton K., Davis S.C., McClatchy D., Valdes P.A., Kanick S.C., Paulsen K.D., et al. Pulsed-light imaging for fluorescence guided surgery under normal room lighting. Opt Lett 2013 Sep; 38(17): 3249-3252, http://dx.doi.org/10.1364/ OL.38.003249.

19. Jacques S.L. How tissue optics affect dosimetry of photodynamic therapy. J Biomed Opt 2010 Sept-Oct; 15(5): 051608, http://dx.doi.org/10.1117/1.3494561.

20. Sandell J.L., Zhu T.C. A review of in-vivo optical properties of human tissues and its impact on PDT. J Biophotonics 2011 Nov; 4(11-12): 773-787, http://dx.doi. org/10.1002/jbio.201100062.

21. Tyrrell J., Thorn C., Shore A., Campbell S., Curnow A. Oxygen saturation and perfusion changes during dermatological methylaminolaevulinate photodynamic therapy. $\mathrm{Br} J$ Dermatol 
2011 Dec; 165(6): 1323-1331, http://dx.doi.org/10.1111/ j.1365-2133.2011.10554.x.

22. Liu B., Farrell T.J., Patterson M.S. A dynamic model for ALA-PDT of skin: simulation of temporal and spatial distributions of ground-state oxygen, photosensitizer and singlet oxygen. Phys Med Biol 2010 Oct; 55(19): 5913-5932, http://dx.doi.org/10.1088/0031-9155/55/19/019.

23. Hamdoon Z., Jerjes W., Upile T., Hopper C. Optical coherence tomography-guided photodynamic therapy for skin cancer: case study. Photodiagnosis Photodyn Ther 2011 Mar; 8(1): 49-52, http://dx.doi.org/10.1016/ j.pdpdt.2010.08.004.

24. Themstrup L., Banzhaf C.A., Mogensen M., Jemec G.B.E. Optical coherence tomography imaging of nonmelanoma skin cancer undergoing photodynamic therapy reveals subclinical residual lesions. Photodiagnosis Photodyn Ther 2014 Mar; 11(1): 7-12, http://dx.doi.org/10.1016/ j.pdpdt.2013.11.003.

25. Mariampillai A., Leung M.K., Jarvi M., Standish B.A., Lee K., Wilson B.C., et al. Optimized speckle variance OCT imaging of microvasculature. Opt Lett 2010 Apr; 35(8): 12571259, http://dx.doi.org/10.1364/OL.35.001257.

26. Zotter S., Pircher M., Torzicky T., Bonesi M., Götzinger E., Leitgeb R.A., Hitzenberger C.K. Visualization of microvasculature by dual-beam phase-resolved Doppler optical coherence tomography. Opt Express 2011; 19(2): 1217-1227, http://dx.doi.org/10.1364/OE.19.001217.

27. Ntziachristos V., Razansky D. Molecular imaging by means of multi-spectral optoacoustic tomography (MSOT). Chem Rev 2010; 110(5): 2783-2794, http://dx.doi.org/10.1021/ cr9002566.

28. Mallidi S., Huang H.C., Liu J.Y., Mensah L., Mai Z., Hasan T. Photoacoustic image guided photodynamic therapy of glioblastoma. Cancer Res 2013 Apr; 73(8 Suppl): 3923, http://dx.doi.org/10.1158/1538-7445.am2013-3923.

29. Balu M., Mazhar A., Hayakawa C.K., Mittal R., Krasieva T.B., König K., et al. In vivo multiphoton NADH fluorescence reveals depth-dependent keratinocyte metabolism in human skin. Biophys J 2013 Jan; 104(1): 258-267, http:// dx.doi.org/10.1016/j.bpj.2012.11.3809.

30. Ulrich M., Klemp M., Darvin M.E., König K., Lademann J., Meinke M.C. In vivo detection of basal cell carcinoma: comparison of a reflectance confocal microscope and a multiphoton tomography. J Biomed Opt 2013 Jun; 18(6): 061229, http://dx.doi.org/10.1117/1.jbo.18.6.061229.

31. König K. Hybrid multiphoton multimodal tomography of in vivo human skin. Intravital 2012 Jul; 1(1): 11-26, http:// dx.doi.org/10.4161/intv.21938. 